Article

\title{
Facile Preparation and Highly Efficient Catalytic Performances of Pd-Cu Bimetallic Catalyst Synthesized via Seed-Mediated Method
}

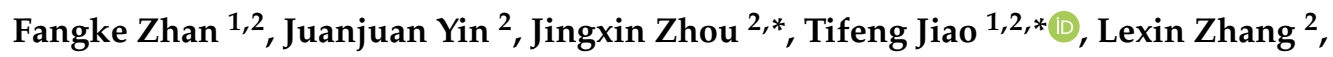 \\ Meirong Xia ${ }^{2}$, Zhenhua Bai ${ }^{3}$ and Qiuming Peng ${ }^{1}$ \\ 1 State Key Laboratory of Metastable Materials Science and Technology, Yanshan University, \\ Qinhuangdao 066004, China; kekelittlebear@126.com (F.Z.); pengqiuming@ysu.edu.cn (Q.P.) \\ 2 Hebei Key Laboratory of Applied Chemistry, School of Environmental and Chemical Engineering, \\ Yanshan University, Qinhuangdao 066004, China; jjy1729@163.com (J.Y.); zhanglexin@ysu.edu.cn (L.Z.); \\ xmr0125@126.com (M.X.) \\ 3 National Engineering Research Center for Equipment and Technology of Cold Strip Rolling, \\ Yanshan University, Qinhuangdao 066004, China; bai_zhenhua@aliyun.com \\ * Correspondence: zhoujingxin@ysu.edu.cn (J.Z.); tfjiao@ysu.edu.cn (T.J.); Tel.: +86-335-806-1569 (J.Z.); \\ $+86-335-805-6854$ (T.J.)
}

Received: 10 November 2019; Accepted: 5 December 2019; Published: 18 December 2019

\begin{abstract}
With the rapid development of industry, the problem of environmental pollution has become increasingly prominent. Exploring and preparing green, efficient, and low cost catalysts has become the key challenge for scientists. However, some conventional preparation methods are limited by conditions, such as cumbersome operation, high energy consumption, and high pollution. Here, a simple and efficient seed-mediated method was designed and proposed to synthesize a highly efficient bimetallic catalyst for catalyzing nitro compounds. A Pd-Cu bimetallic composite (BCM) can be prepared by synthesizing the original seed crystal of precious metal palladium, then growing the mature nanocrystalline palladium and supporting the transition metal copper. Importantly, after eight consecutive catalytic cycles, the conversion of the catalyzed 2-NA was $84 \%$, while the conversion of the catalyzed 4-NP was still 72\%. And the catalytic first order rates of 2-NA and 4-NP constants were $0.015 \mathrm{~s}^{-1}$, and $0.069 \mathrm{~s}^{-1}$, respectively. Therefore, current research of nanocomposites catalyst showed great significance for serious environmental pollution problems and the protection of living environment, providing a new idea for the preparation of new bimetallic catalytic materials.
\end{abstract}

Keywords: seed-mediated; bimetallic catalyst; nanocomposites; catalyst

\section{Introduction}

With the rapid development of modern society, the problem of environmental pollution has become increasingly serious, and the types and quantities of chemical pollutants in the atmosphere and soil have increased rapidly. In particular, any discharge of chemical dyes poses a serious threat to human health. In recent years, how to obtain efficient green, low-cost, recyclable catalysts has become a key and difficult point in this field. Some researchers have developed a variety of different methods to deal with chemical dyes, such as adsorption degradation [1,2], electrocatalytic oxidation [3], biodegradation $[4,5]$ and photocatalytic degradation, etc. [6-8].

In addition to the above methods, endless streams of catalyst materials have been developed, such as p-nitrophenol (4-NP) and o-nitroaniline (2-NA) [9-13], in order to solve these compounds which pose a major hazard to the environment and human health. In addition, a common catalyst for palladium is well-known [14,15]. In recent years, some Pd-doped composite catalyst materials have 
been reported. Palladium has a very good catalytic activity for the coupling reaction of carbon-carbon bonds due to its strong affinity for hydrogen, and its cost advantage is superior to other metals such as platinum. At the same time, palladium can be used in the Suzuki reaction [16,17], purification storage and detection $[18,19]$ and dye catalysis $[20,21]$. Due to high electrical conductivity and thermal conductivity of transition metals, and because the cost is much lower compared to precious metals, special attention to copper metal has continued to increase [22-26]. Therefore, more and more composite nanomaterials of two metals, palladium and copper, have appeared in recent years. Iwamoto et al. successfully prepared a nitrate-removed nitrate from brine by using an anion exchange resin (AIER) as a carrier [27]. Ye et al. have prepared Pd-Cu nanoparticles supported on reduced graphene oxide nanosheets with high catalytic performance for two-step reduction [28]. Mao et al. designed and obtained well-dispersed $\mathrm{Pd}-\mathrm{Cu}$ bimetallic nanocrystals, which showed good performance in both ethanol electrooxidation and tire peroxidation [29-32]. However, the above reports may have cumbersome preparations, adverse effects such as resource consumption and environmental pollution. In this work, a uniform, low-cost, green, non-polluting bead-like palladium particle was prepared by seed-mediated method, which overcomes particle agglomeration due to the large surface energy. After the copper is loaded, the shape can still be clearly controlled. Other than that, synthesizing structurally defined bimetallic NPs has been a great challenge for a long time. But the seed-mediated method in this study used uniform Pd seed nanocrystals as the cores when growth Pd nanocrystals, excluded large-scale heteronuclear crystals generated and spontaneous nucleation firstly. Secondly, the second metal is loaded on the premise that the Pd nanocrystals are still highly homogeneous. Compared with the one-step methodology, the size distribution of the particles is smaller, and the preparation process is simpler and easier to control [33]. The novel $\mathrm{Pd}-\mathrm{Cu}$ bimetallic composite (BCM) catalyst prepared by the method has excellent catalytic degradation performance for p-nitrophenol (4-NP) and o-nitroaniline (2-NA). The current research work provides a broad application prospect for palladium-based bimetallic composite materials in the field of chemical dye catalytic degradation and sewage treatment.

\section{Materials and Methods}

\subsection{Materials}

Ascorbic acid (AA), palladium (II) chloride ( $\left.\mathrm{PdCl}_{2}, 59-60 \%\right), \mathrm{HCl}$ solution, potassium iodide (KI, 99\%) purchased from Aladdin Reagent (Shanghai, China). Copper (II) chloride $\left(\mathrm{CuCl}_{2}, 98 \%\right.$ ), and sodium dodecyl benzene sulfonate (SDBS) $(90 \%)$ were purchased from Sigma Aldrich (St. Louis, MO, USA). Ethanol $\left(\mathrm{C}_{2} \mathrm{H}_{5} \mathrm{OH}\right.$, analytical reagent) was supplied by Tianjin Guangfu Fine Chemical Research Institute (Tianjin, China), 2-nitroaniline (2-NA, 99\%), and 4-Nitrophenol (4-NP, 99\%) were obtained from Alfa Aesar (Beijing, China). Ultra-pure water was obtained by a Milli-Q Millipore filter system (Millipore Co., Ltd., Bedford, MA, USA) with a resistivity of $18.2 \mathrm{M} \Omega \mathrm{cm}^{-1}$. All chemicals were used as received without purification.

\subsection{Fabrication of $P d-C u$ Bimetallic Catalyst}

The $10 \mathrm{mM} \mathrm{H}_{2} \mathrm{PdCl}_{4}$ solution was obtained by adding $0.1773 \mathrm{~g}$ of $\mathrm{PdCl}_{2}$ in $10 \mathrm{~mL}$ of $0.2 \mathrm{M} \mathrm{HCl}$ solution and then moved into the volumetric flask before diluting to $100 \mathrm{~mL}$ ultra-pure water.

\subsection{Synthesis of Palladium Seeds}

The preparation of palladium was obtained according to the following method. Specifically, $20 \mathrm{~mL}$ of $12.5 \mathrm{mM} \mathrm{CTAB}$ solution was added to $1 \mathrm{~mL}$ of $10 \mathrm{mM} \mathrm{H}_{2} \mathrm{PdCl}_{4}$ solution heated at $95^{\circ} \mathrm{C}$ under stirring. After $5 \mathrm{~min}$, as the mixture was mixed into the solution, $160 \mu \mathrm{L}$ of freshly prepared $100 \mathrm{mM}$ AA solution was added quickly, then the mixture was inverted once, and then to avoid interference, the reaction was allowed to carry on for $30 \mathrm{~min}$. The solution, filled with Pd seed, was stored at $30^{\circ} \mathrm{C}$ for future use as seeds. 


\subsection{Seed-Mediated Growth of Bead-Chain Palladium Nanocrystals}

In a typical synthesis, $25 \mu \mathrm{L}$ of $1 \mathrm{mM}$ KI solution was added to $5 \mathrm{~mL}$ of $100 \mathrm{mM}$ SDBS solution kept at $40{ }^{\circ} \mathrm{C}, 80{ }^{\circ} \mathrm{C}, 90{ }^{\circ} \mathrm{C}$, respectively, $125 \mu \mathrm{L}$ portion of $10 \mathrm{mM} \mathrm{H}_{2} \mathrm{PdCl}_{4}$ solution and $40 \mu \mathrm{L}$ of seed palladium solution as synthesized were then added. Finally, $100 \mu \mathrm{L}$ of freshly prepared $100 \mathrm{mM}$ ascorbic acid (AA) solution was added by drop, finally the solution was mixed thoroughly. The resulting solution was sited in a water bath for $12,1,0.5 \mathrm{~h}$ corresponding to the reaction temperature. The reactions were stopped by centrifugation $(8000 \mathrm{rpm}, 10 \mathrm{~min})$. The precipitates was diluted and dispersed with $3 \mathrm{~mL}$ of water.

\subsection{Synthesis of $P d-C u$ Nanomaterial}

For the part of Pd-Cu nanomaterial growth, $2 \mathrm{~mL}$ aqueous SDBS $(0.2 \mathrm{M})$ solution was added to the solution which has mixed $0.025 \mathrm{~mL} \mathrm{H}_{2} \mathrm{PdCl}_{4}(10 \mathrm{mM})$ solution and $0.05 \mathrm{~mL}$ of $\mathrm{CuCl}_{2}(10 \mathrm{mM})$ aqueous solution. What has to be aware of is that the preparation of the $\mathrm{CuCl}_{2}$ solution needs a small amount of hydrochloric acid solution to adjust the $\mathrm{pH}$ value. Following with $1.5 \mathrm{~mL} \mathrm{AA}(0.1 \mathrm{M})$ aqueous solution, the $21.4 \mathrm{~mL}$ of water was added, and then $1 \mathrm{~mL}$ solution containing the Pd nanocrystal was added. The vials was capped, inverted once, and allowed to sit undisturbed in a $25{ }^{\circ} \mathrm{C}$ water bath $12 \mathrm{~h}$. The product was collected by centrifugation of the solution at $8000 \mathrm{rpm}$ for 20minutes; the supernatant was removed, and the final products were dispersed in ultrapure water and ethanol for UV-vis extinction spectra characterization. Two more centrifugations ( $8000 \mathrm{rpm}, 10 \mathrm{~min})$ were applied to the final product for XRD characterization, transmission electron microscope (TEM), and scanning electron microscopy (SEM).

\subsection{Catalytic Performance Test}

The 2-NA or 4-NP $(2 \mathrm{~mL}, 5 \mathrm{mM})$ solution was added to a freshly prepared $\mathrm{NaBH}_{4}(20 \mathrm{~mL}, 0.01 \mathrm{M})$ solution at room temperature. In this case, $\mathrm{NaBH}_{4}$ is a reducing agent throughout the reduction reaction. $1 \mathrm{mg}$ of the Pd-Cu bimetallic composite catalyst was added to $2 \mathrm{~mL}$ of ethanol to form a stable $0.5 \mathrm{mg} / \mathrm{mL}$ suspension. A portion of suspension $(2 \mathrm{~mL}, 0.5 \mathrm{mg} / \mathrm{mL})$ was then added to the 2-NA or 4-NP solution for catalytic reduction, and the whole process was tested at room temperature and under UV spectrum. The Pd-Cu bimetallic composite catalyst was recycled in the next cycle experiment before washed 3 times by the ultrapure water and ethanol. The same solid powder was repeated use along with fresh 2-NA or 4-NP and $\mathrm{NaBH}_{4}$ aqueous solutions.

\subsection{Characterization}

The synthesized materials were treated to remove water by freeze drying at a temperature of $-48{ }^{\circ} \mathrm{C}$ for over $24 \mathrm{~h}$ via a FD-1C-50 instrument (Beijing Boyikang Experimental Instrument Co., Ltd., Beijing, China). TEM studies were performed with a transmission electron microscopy (TEM, HT7700, Hitachi high technologies Corporation, Ibaraki, Japan). The structures of Pd-Cu nanomaterials were investigated via scanning electron microscope (SEM) Field Emission Gun FEI QUANTA FEG 250 (FEI Corporate, Hillsboro, OR, USA). X-ray diffraction (XRD) were obtained with an X-ray diffractometer equipped with a Bragg diffraction setup (SMART LAB, Rigaku, Akishima, Japan) and a Cu K $\alpha$ X-ray radiation source to further characterize the obtained materials. High-resolution transmission electron microscopy (HRTEM, Tecnai-G2 F30 S-TWIN, Philips, Eindhoven, Netherlands) images were acquired with a JEM-2010 electron microscope (Hitachi, Tokyo, Japan) operated at $200 \mathrm{kV}$. X-ray photoelectron spectroscopy (XPS) was performed using a Bragg diffraction setup (ESCALAB 250Xi XPS) with Al K $\alpha$ $\mathrm{X}$-ray source. 


\section{Results and Discussion}

\subsection{Structural Characterization of Pd-Cu Nanocomposites}

In this work, Pd-Cu bimetallic composite materials (BCM) synthesized by Seed-mediated synthetic method shows homogeneous appearance and high catalytic performance. First, a solution of $\mathrm{H}_{2} \mathrm{PdCl}_{4}$ $(1 \mathrm{~mL}, 10 \mathrm{mM})$ was added to SDBS solution $(20 \mathrm{~mL}, 12.5 \mathrm{mM})$, and then kept the mixture at $95^{\circ} \mathrm{C}$ for 5 min. As shown in Figure 1a, as the AA solution was added, the $\mathrm{Pd}^{2+}$ ions were reduced to form Pd seed NPs. Through adding Pd seed into growth solution containing $\mathrm{I}^{-}$, SDBS and the reducing agent fresh AA solution, Pd atoms begin to deposit on Pd seeds NPs. Bakshi has reported that the correlation between sodium dodecyl sulfate (SDS) and its ability in designing nano-morphologies. Therefore, it can be inferred that the SDBS, which was a kind of anionic surfactant, which is the same as sodium dodecyl sulfate (SDS), was used to form a dense layer. And the dense layer induces the growth of palladium particles on one crystal plane and restricts the growth on other crystal faces, then the bead-chain palladium nanocrystals has been given [32]. And adding $\mathrm{I}^{-}$ions could avoid the structure fluctuations of the palladium nanoparticles and depress the spontaneous nucleation of it. That can be shown in detail by Niu et al. [33]. A set of comparative tests was set with the preparation Pd nanocrystals, and the phenomenon of spontaneous nucleation was obvious with absence of $\mathrm{I}^{-}$[33]. Therefore, it is very important to add halogen elements iodine to generate a complete palladium crystal. Then, the seed-mediated method was also used in the step of palladium nanocrystals composite copper particles as shows in Figure 1b. At this time, palladium nanocrystals were added as seeds to the growth solution containing $\mathrm{Cu}^{2+}$ ions, and $\mathrm{Cu}^{2+}$ ions were uniformly reduced on the beads of palladium to form the bead chain structure. In order to prevent copper from being oxidized by oxygen in solution and air during the reduction process, the whole process requires nitrogen protection in a slow reduction process. Next, the catalytic activity of $\mathrm{Pd}-\mathrm{Cu}$ bimetallic composite materials (BCM) for 2-NA and 4-NP was studied as Figure 1c performed. In this part, the catalytic activity for the functional group $-\mathrm{NO}_{2}$ is attributed to the mutual synergy of $\mathrm{Pd}-\mathrm{Cu}$ bimetallic composite materials (BCM). First, when $\mathrm{BH}_{4}{ }^{-}$is added to the solution containing 4-NP, electrons are transferred from the $\mathrm{BH}_{4}{ }^{-} \mathrm{donor}^{-}$ to the 4-NP receptor, and then amino derivative has been produced. After adding the catalyst, the amino derivative and $\mathrm{BH}_{4}{ }^{-}$are adsorbed on the surface of $\mathrm{Pd}-\mathrm{Cu}$ bimetallic composite materials (BCM) by the combination of hydrogen bonding and physical adsorption. When electrons transfer Pd-Cu NPs, the hydride forms a hydrogen atom and spontaneously undergoes a reduction reaction on the surface of Pd-Cu bimetallic composite materials (BCM). Finally, the prepared 4-nitroaniline (4-AP) was desorbed from the surface of the catalyst [34]. Similarly, the nitro group in the 2-NA molecule should be converted to an amino group under conditions of catalytic hydrogenation (Figure 1d) [35].

After adjusting the reaction temperature and the corresponding reaction time, the most suitable products $\left(80^{\circ} \mathrm{C}, 1 \mathrm{~h}\right)$ are selected, which has the most clear and homogeneous morphology as well as the better performance. To further characterize the morphology of the composite, a TEM test was performed as shown in Figure 2. As can be seen from Figure 2a, the prepared palladium particles are heaped together as many strings of irregular beads. They grow into uniform beads under the action of the growth liquid and the halogen element $\mathrm{I}^{-}$ion, which depress the spontaneous nucleation of $\mathrm{Pd}$ atoms when they deposition on the Pd seed nanocrystal [33]. Finally, a uniform bead chain shape is formed. In Figure 2b, the reduced composite of copper causes the diameter of some of the beads on the bead chain to become larger and the color to become significantly darker. In addition, in order to further observe the structure of the prepared Pd-Cu bimetal composite (BCM), high-resolution transmission electron microscopy (HRTEM) analysis is necessary, as shown in Figure 2c. Compared to the TEM image of palladium, the image in Figure 2a clearly shows many beads that loaded the copper atom in the chain are darker than the original pure palladium beads. Obviously, the obtained copper and palladium show a clear crystal structure and a distinct lattice fringe. The plane spacing of the lattice data of copper $\mathrm{d}(111)$ is $0.217 \mathrm{~nm}$, while the plane spacing $\mathrm{d}(111)$ of the lattice data of palladium is $0.223 \mathrm{~nm}[36,37]$. Importantly, the reported palladium nanocrystals have a clear and 
distinct morphology, and very uniform dispersion compared to other reports [38-41]. At the same time, the shape of the bead chain is also very intuitive, and the shape after loading the copper is still very clear. More notably, the palladium and copper molar ratio selected in this report is more suitable for the preparation of homogeneous appearance of $\mathrm{Pd}-\mathrm{Cu}(\mathrm{BCM})$ compared with other research reports [42]. In addition, as shown in Figure 3, the results of elemental map analysis indicated that the prepared Pd-Cu bimetallic composite materials (BCM) were mainly composed of Pd and Cu elements. These elemental diagrams further suggested that copper was supposed to be reduced on the palladium nanocrystals with the uniform dispersion, which needed to be confirmed by further studies.
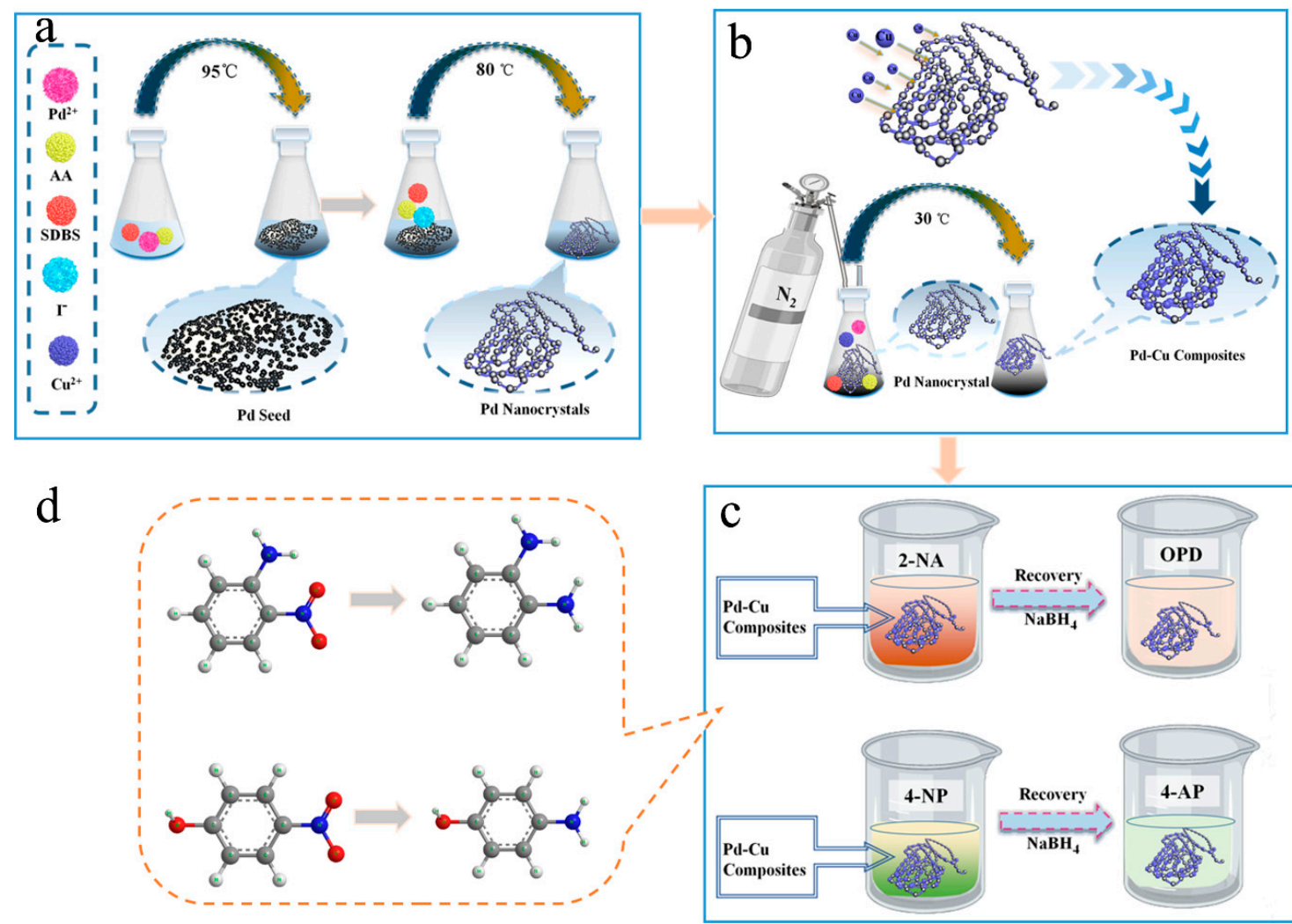

Figure 1. Schematic illustration of preparation as well as catalyt ic application of Pd-Cu bimetallic composite materials (BCM): prepared Pd seed and Pd nanocrystals (a); prepared Pd-Cu composites (b); catalytic reduction test of Pd-Cu composites (c); ball-and-stick molecular model of 2-NA, OPD and 4-NP,4-AP, respectively $(\mathbf{d})$.

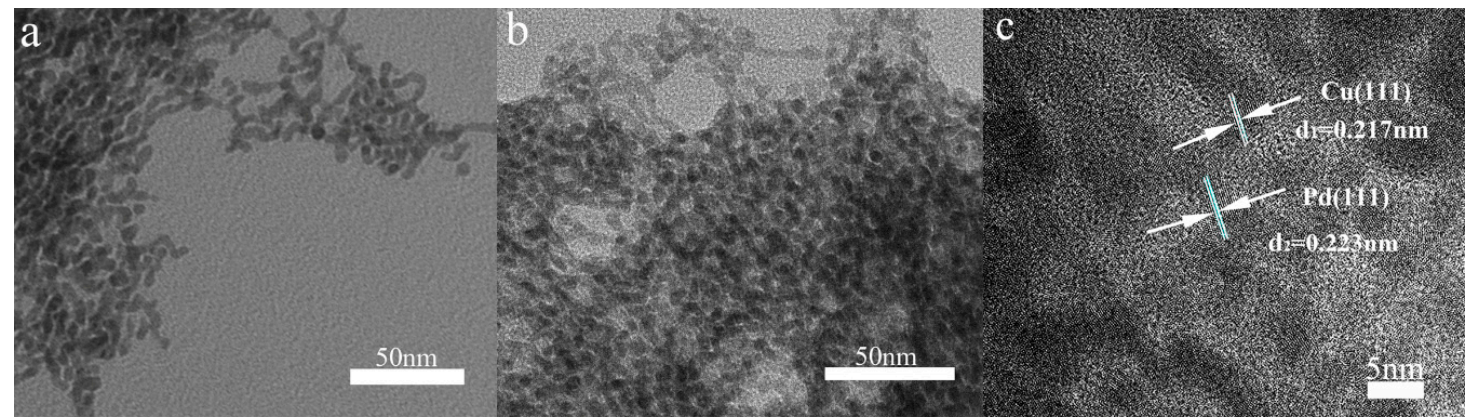

Figure 2. TEM images of pure Pd sample (a), Pd-Cu bimetallic composite (BCM) sample (b) and HTEM image of Pd-Cu bimetallic composite (c). 

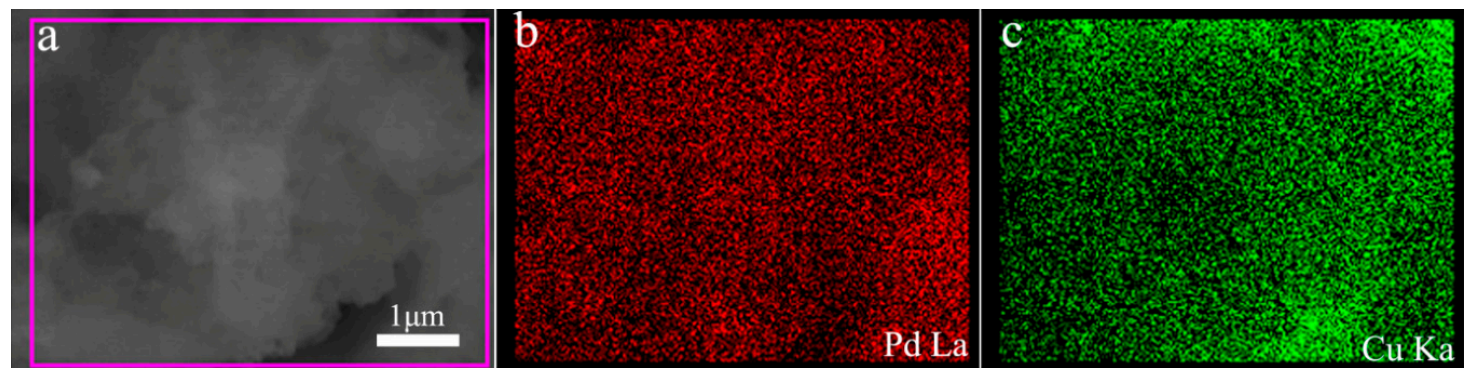

Figure 3. SEM image and of Pd-Cu bimetallic composite nanoparticles (a) with $\mathrm{Pd} / \mathrm{Cu}$ elemental mapping $(\mathbf{b}, \mathbf{c})$.

XPS testing of the obtained Pd-Cu bimetallic composite (BCM) for more detailed analysis is of great significance for studying the elemental composition and chemical state of the composite, as shown in Figure 4 [43]. The Pd 3d region shows the ratio of the bimodal binding energy of the spin coupling to the metal Pd in the expected 2:3 by high resolution scanning (Figure $4 \mathrm{~b}$ ). After the composite copper, the content of the main $\mathrm{Cu}^{0}(67.5 \%)$ in the composite material is much larger than that of $\mathrm{Cu}^{2+}(32.5 \%)$, indicating that the $\mathrm{Cu}$ metal component is successfully reduced to the obtained composite material, as shown in Figure 4c [44]. Here, there is a small amount of $\mathrm{Cu}^{2+}$ present, probably because the $\mathrm{Cu}^{2+}$ added during the experiment is excessive.
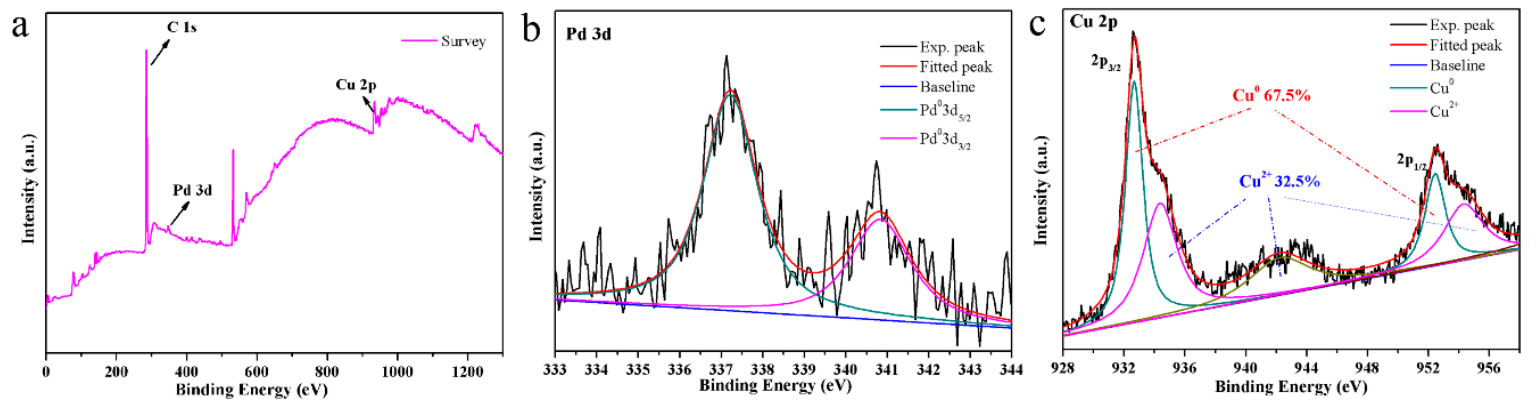

Figure 4. (a) wide XPS spectrum of the Pd-Cu bimetallic composite materials (BCM) samples; (b) Pd3d peaks; (c) Cu2p peaks.

\subsection{Catalytic Performance of $\mathrm{Pd}-\mathrm{Cu}$ Nanocomposites}

Next, the obtained $\mathrm{Pd}-\mathrm{Cu}$ bimetallic composite (BCM) was used as a catalyst to study its catalytic performance for the nitro compound (4-NP, 2-NA) [45-53]. As shown in Figure 5, the catalytic performance of $\mathrm{Pd}-\mathrm{Cu}$ bimetallic composite (BCM) for 2-NA reduction was investigated. As shown in Figure $5 \mathrm{a}$, after the addition of fresh $\mathrm{NaBH}_{4}$ to the 2-NA solution, the absorption peak of the mixed solution showed no significant change. After adding the Pd-Cu bimetallic composite (BCM) catalyst for $30 \mathrm{~min}$, the 2-NA of the solution was completely reduced to o-phenylenediamine (OPD). Experiments show that the experimentally added $\mathrm{Pd}-\mathrm{Cu}$ catalyst has a strong catalytic ability for the reduction of 2-NA.

The catalytic activity of the synthesized $\mathrm{Pd}-\mathrm{Cu}$ bimetallic composite (BCM) was evaluated by catalytic reduction of p-nitrophenol with a $\mathrm{NaBH}_{4}$ solution. As shown in Figure 5 d, when $\mathrm{NaBH}_{4}$ was not added, the 4-NP solution was pale yellow and the absorption peak was $317 \mathrm{~nm}$. After the addition, the solution changed from light yellow to bright yellow due to the large amount of p-nitrophenol ions, and the absorption peak shifted to $400 \mathrm{~nm}$. The absorption peak of 4-AP then appeared near $295 \mathrm{~nm}$, at which time the solution became colorless. The pseudo-first order equation of kinetics can be used to assess the rate of catalytic reduction reactions. The absorbance of 2-NA is proportional to the concentration of the solution, so we can describe the catalytic reduction by a linear relationship between $\ln \left(C_{t} / C_{0}\right)\left(C_{t}\right.$ represents the concentration of the mixed solution at time $t$, and $C_{0}$ represents 
the initial concentration) And the change law of reaction time $t$. The kinetics of the reduction reaction is shown in Figure 5f, and its linear relationship is consistent with the first kinetic equation. The reaction rate constant $\mathrm{k}$ is fitted to the pseudo-level $\mathrm{k}$ of $\mathrm{O}$-nitrophenol catalyzed by $\mathrm{Pd}-\mathrm{Cu}$ bimetallic composite (BCM), and the calculated value is $0.015 \mathrm{~s}^{-1}$. In addition, as a comparison, the reaction rate constant $\mathrm{k}\left(0.069 \mathrm{~s}^{-1}\right)$ fits the pseudo first-order reaction of $\mathrm{Pd}-\mathrm{Cu}$ bimetallic composite (BCM) catalyst to p-nitrophenol. Obviously, Pd-Cu bimetallic composite (BCM) has a better catalytic effect on 2-NA.
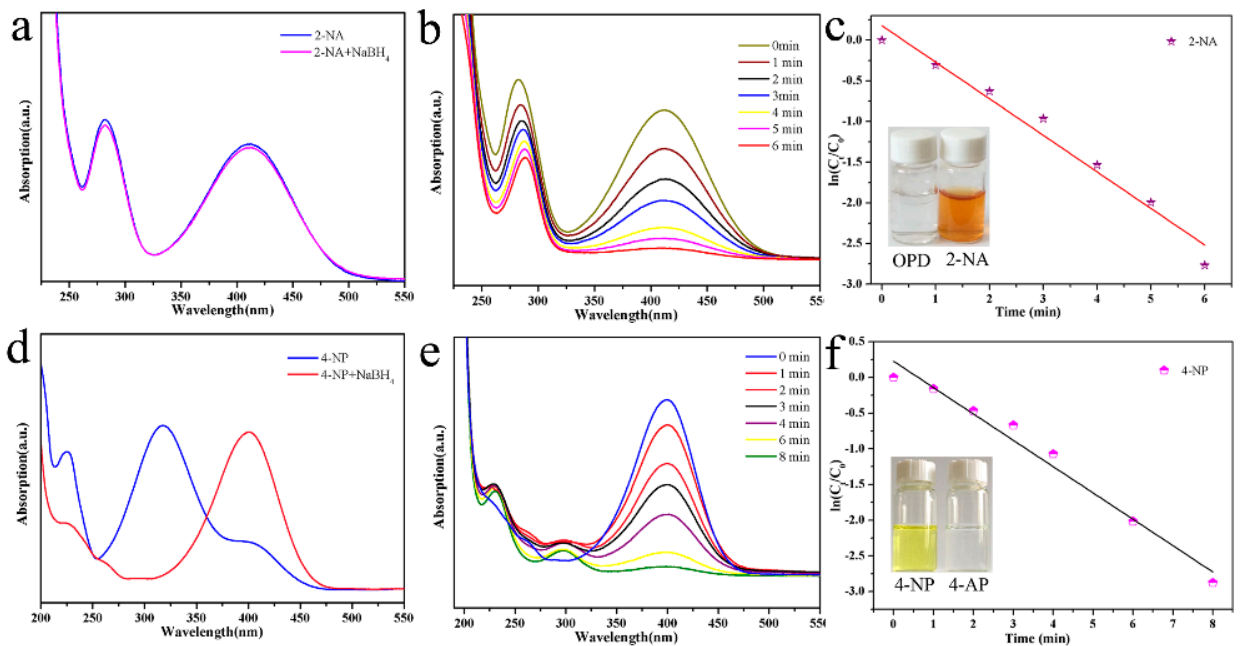

Figure 5. Catalytic reduction test of 2-NA transforming to o-phenylenediamine (OPD) (a,b) and 4-NP transforming to 4-AP (d,e) by Pd-Cu bimetallic composite materials (BCM) samples; and relationship between $\ln \left(C_{t} / C_{0}\right)$ and the reaction time $(t)$ of reduction catalyst $(c, f)$.

In order to compare the superiority of the Pd-Cu bimetallic composite catalyst, a series of comparative tests were conducted. Figure 6 is a graph of the results of the catalyzing 2-NA and 4-NP by pure palladium nanocrystals, prepared by the same seed-mediated method. It can be seen from Figure $6 \mathrm{a}$ that the pure palladium nanocrystal catalyzed the completion of the 2-NA catalytic reduction reaction for $39 \mathrm{~min}$, which showed relatively weak results compared with the $\mathrm{Pd}-\mathrm{Cu}$ bimetallic composite catalyst catalyzing 2-NA. Similarly, the completion time of the 4-NP reduction reaction catalyzed by pure palladium nanocrystals was $45 \mathrm{~min}$, which showed relatively weak results compared with the Pd-Cu bimetallic composite catalyst catalyzing 4-NP. Therefore, it can be concluded that the $\mathrm{Pd}-\mathrm{Cu}$ bimetallic composite catalyst has excellent catalytic effects on 2-NA and 4-NP.
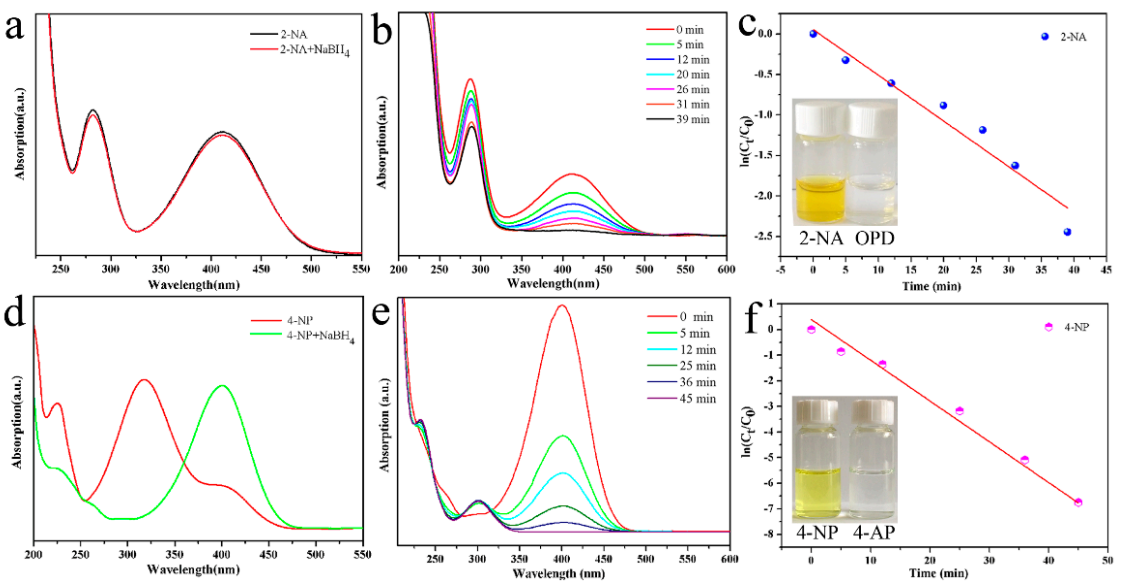

Figure 6. Catalytic reduction test of 2-NA transforming to $\mathrm{OPD}(\mathbf{a}, \mathbf{b})$ and4-NP transforming to 4-AP $(\mathbf{d}, \mathbf{e})$ 2-NA,4-NP catalytic comparison experiment by pure palladium; and relationship between $\ln \left(\mathrm{C}_{\mathrm{t}} / \mathrm{C}_{0}\right)$ and the reaction time $(\mathrm{t})$ of reduction catalyst $(\mathbf{c}, \mathbf{f})$. 
In addition, the widely-used catalyst should have good recyclability and excellent stability. To this end, eight consecutive catalytic experiments were repeated using freshly prepared 4-NP and 2-NA solutions, as shown in Figure 7. The results showed that the stability of the catalyzed 2-NA and 4-NP remained at $84 \%$, and $72 \%$, respectively, after eight cycles. Therefore, the synthesized Pd-Cu bimetallic composite (BCM) has excellent catalytic and stability for catalyzing 2-NA and 4-NP. The catalytic efficiency is slightly reduced due to the loss of nanoparticles during the washing process. In view of the above results, the new Pd-Cu bimetallic composite (BCM) provides analysis for new catalyst fields, such as dye catalytic degradation and sewage treatment, and opens up a new path for the research of palladium series materials and self-assembled composites [54-64].
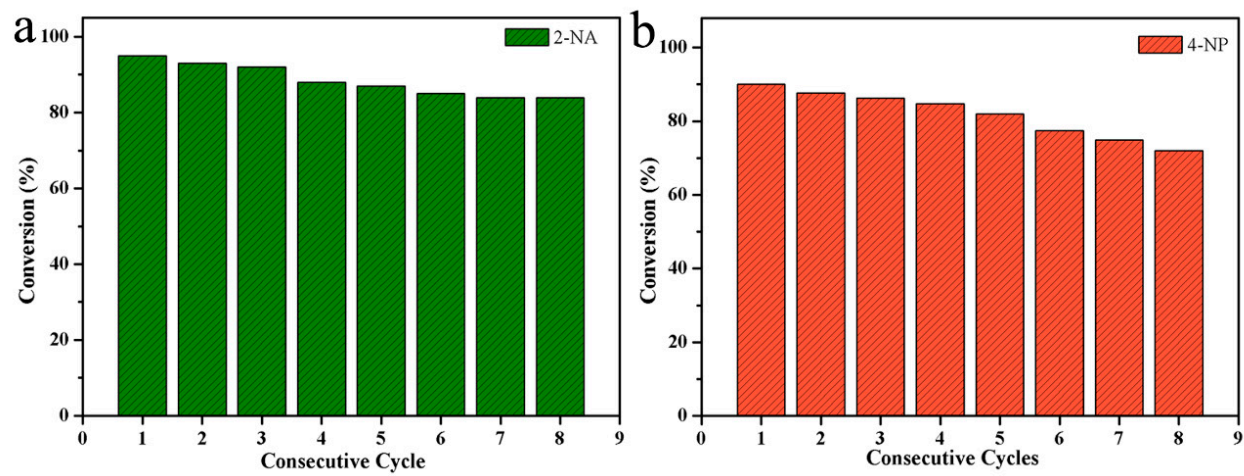

Figure 7. The cyclic catalytic capacity of Pd-Cu bimetallic composite materials (BCM) composite for the reduction of 2-NA (a), 4-NP (b).

\section{Conclusions}

In summary, in this work, a homogeneous bead chain Pd-Cu bimetallic composite (BCM) catalyst was proposed to prepare by a seed-mediated method. In particular, the morphology of the bimetallic catalyst can be significantly controlled by sodium dodecyl benzene sulfonate blocking agent and halogen element iodine. The pre-pressed Pd-Cu bimetallic composite (BCM) catalyst exhibits excellent reducing power for the nitro compounds 2-NA and 4-NP. In addition, after eight consecutive cycles of the catalyst, the experimental results showed that the conversion rate of the catalytic 2-NA is still as high as $84 \%$. Interestingly, the bimetallic catalyst showed high catalytic activity and stability. Therefore, this study provides a new method for the sustainable study of new precious metal-transition metal bimetallic catalysts.

Author Contributions: T.J. and J.Z. conceived and designed the experiments; F.Z., J.Y., and J.Z. performed the experiments; L.Z. and M.X. analyzed the data; Z.B. and Q.P. contributed reagents/materials/analysis tools; J.Y., F.Z., and T.J. wrote and revised the paper. All authors have read and agreed to the published version of the manuscript.

Funding: This research was funded by the National Natural Science Foundation of China (no. 21872119), the Support Program for the Top Young Talents of Hebei Province, the China Postdoctoral Science Foundation (no. 2015M580214), and the Research Program of the College Science and Technology of Hebei Province (no. ZD2018091).

Conflicts of Interest: The authors declare no conflict of interest.

\section{References}

1. Ma, W.; Fang, Y. Experimental (SERS) and theoretical (DFT) studies on the adsorption of p-, m-, and o-nitroaniline on gold nanoparticles. J. Colloid Interface Sci. 2006, 303, 1-8. [CrossRef]

2. Shojaat, R.; Saadatjoo, N.; Karimi, A.; Aber, S. Simultaneous adsorption-degradation of organic dyes using $\mathrm{MnFe}_{2} \mathrm{O}_{4}$ /calcium alginate nano-composites coupled with GOx and Laccase. J. Environ. Chem. Eng. 2016, 4, 1722-1730. [CrossRef]

3. Zahorulko, S.; Shmychkova, O.; Luk'yanenko, T.; Dmitrikovab, L.; Velichenkoa, A. The comparative study of electrocatalytic activity of various anode materials in respect to the oxidation of nitroanilines. Mater. Today 2019, 6, 242-249. [CrossRef] 
4. Yan, Z.; Xuewei, H.; Xuan, L.; Weiqing, S.; Min, Y. Selective bioaugmentation treatment for coexistence wastewater containing-toluidine and easily degradable substrates. Ind. Water Treat. 2014, 35, 101-102.

5. Reemtsma, T.; Jakobs, J. Concerted Chemical and Microbial Degradation of Sulfophthalimides Formed from Sulfophthalocyanine Dyes by White-Rot Fungi. Environ. Sci. Technol. 2001, 35, 4655-4659. [CrossRef] [PubMed]

6. Guillar, C.; Lachheb, H.; Houas, A.; Ksibi, M.; Elaloui, E.; Herrmann, J.M. Influence of chemical structure of dyes, of $\mathrm{pH}$ and of inorganic salts on their photocatalytic degradation by $\mathrm{TiO}_{2}$ comparison of the efficiency of powder and supported $\mathrm{TiO}_{2}$. J. Photochem. Photobiol. A 2003, 158, 27-36. [CrossRef]

7. Khataee, A.R.; Kasiri, M.B. Photocatalytic degradation of organic dyes in the presence of nanostructured titanium dioxide: Influence of the chemical structure of dyes. J. Mol. Catal. A 2010, 328, 27-36. [CrossRef]

8. Yousefi, S.R.; Ghanbari, D.; Salavati-Niasari, M.; Hassanpour, M. Photo-degradation of organic dyes: Simple chemical synthesis of $\mathrm{Ni}(\mathrm{OH})_{2}$ nanoparticles, $\mathrm{Ni} / \mathrm{Ni}(\mathrm{OH})_{2}$ and $\mathrm{Ni} / \mathrm{NiO}$ magnetic nanocomposites. J. Mater. Sci. 2015, 27, 1-10. [CrossRef]

9. Zhao, B.; Mele, G.; Pio, I.; Li, J.; Palmisano, L.; Vasapollo, G. Degradation of 4-nitrophenol (4-NP) using $\mathrm{Fe}-\mathrm{TiO}_{2}$ as a heterogeneous photo-Fenton catalyst. J. Hazard. Mater. 2010, 176, 569-574. [CrossRef]

10. Marais, E.; Nyokong, T. Adsorption of 4-nitrophenol onto Amberlite ${ }^{\circledR}$; IRA-900 modified with metallophthalocyanines. J. Hazard. Mater. 2008, 152, 293-301. [CrossRef]

11. Kampouri, S.; Nguyen, T.N.; Spodaryk, M.; Palgrave, R.G.; Züttel, A.; Smit, B.; Stylianou, K.C. Concurrent Photocatalytic Hydrogen Generation and Dye Degradation Using MIL-125- $\mathrm{NH}_{2}$ under Visible Light Irradiation. Adv. Funct. Mater. 2018, 28, 1806368. [CrossRef]

12. Farooqi, Z.H.; Naseem, K.; Begum, R.; Ijaz, A. Catalytic Reduction of 2-Nitroaniline in Aqueous Medium Using Silver Nanoparticles Functionalized Polymer Microgels. J. Inorg. Organomet. Polym. Mater. 2015, 25, 1554-1568. [CrossRef]

13. Peng, Z.; Li, Y.; Feng, W.; Zhao, Q.; Min, L.; Jia, S. Adsorption behavior of XDA-1 resin on o-nitroaniline and its application. Ind. Water Treat. 2017, 8, 7085-7093.

14. Adams, B.D.; Chen, A. The Role of Palladium in a Hydrogen Economy. Mater. Today 2011, 14, $282-289$. [CrossRef]

15. Zahmakıran, M.; Özkar, S. Metal Nanoparticles in Liquid Phase Catalysis: From Recent Advances to Future Goals. Nanoscale 2011, 3, 3462-3481. [CrossRef]

16. Pérezlorenzo, M. Palladium nanoparticles as efficient catalysts for Suzuki cross-coupling reactions. J. Phys. Chem. Lett. 2012, 3, 167-174. [CrossRef]

17. Torborg, C.; Beller, M. ChemInform Abstract: Recent Applications of Palladium-Catalyzed Coupling Reactions in the Pharmaceutical, Agrochemical, and Fine Chemical Industries. Adv. Synth. Catal. 2009, 351, 3027-3043. [CrossRef]

18. Ju, S.P.; Weng, M.H.; Huang, W.C. Potential applications of two ultrathin Pd nanowires to the hydrogen economy. J. Mater. Chem. 2012, 22, 20319. [CrossRef]

19. Cooney, D.A.; Way, J.D.; Wolden, C.A. A comparison of the performance and stability of Pd/BCC metal composite membranes for hydrogen purification. Int. J. Hydrog. Energy 2014, 39, 19009-19017. [CrossRef]

20. Esumi, K.; Isono, R.; Yoshimura, T. Preparation of PAMAM- and PPI-metal (silver, platinum, and palladium) nanocomposites and their catalytic activities for reduction of 4-nitrophenol. Langmuir 2004, 20, 237-243. [CrossRef]

21. Jia, L.; Zhang, W.; Xu, J.; Cao, J.; Xu, Z.; Wang, Y. Facile Fabrication of Highly Active Magnetic Aminoclay Supported Palladium Nanoparticles for the Room Temperature Catalytic Reduction of Nitrophenol and Nitroanilines. Nanomaterials 2018, 8, 409. [CrossRef] [PubMed]

22. Wu, N.L.; Lee, M.S. Enhanced $\mathrm{TiO}_{2}$ photocatalysis by $\mathrm{Cu}$ in hydrogen production from aqueous methanol solution. Int. J. Hydrog. Energy 2004, 29, 1601-1605. [CrossRef]

23. Xu, S.; Ng, J.; Zhang, X.; Bai, H.; Sun, D.D. Fabrication and comparison of highly efficient Cu incorporated $\mathrm{TiO}_{2}$ photocatalyst for hydrogen generation from water. Int. J. Hydrog. Energy 2010, 35, 5254-5261. [CrossRef]

24. Dhas, N.A.; Raj, C.P.; Gedanken, A. Synthesis, characterization, and properties of metallic copper nanoparticles. Chem. Mater. 1998, 10, 1446-1452. [CrossRef]

25. Vitulli, G.; Bernini, M.; Bertozzi, S.; Pitzalis, E.; Salvadori, P.; Coluccia, S.; Martra, G. Nanoscale copper particles derived from solvated $\mathrm{Cu}$ atoms in the activation ofmolecular oxygen. Chem. Mater. 2002, 14, 1183-1186. [CrossRef] 
26. Liu, Z.; Bando, Y. A novel method for preparing copper nanorods and nanowires. Adv. Mater. 2003, 15, 303-305. [CrossRef]

27. Iwamoto, M.; Tanaka, Y. Preparation of metal ion-planted mesoporous silica by template ion-exchange method and its catalytic activity for asymmetric oxidation of sulfide. Catal. Serv. Jpn. 2001, 5, 25-36. [CrossRef]

28. Ye, H.; Li, Y.; Chen, J.; Sheng, J.; Fu, X.Z.; Sun, R.; Wong, C.P. PdCu alloy nanoparticles supported on reduced graphene oxide for electrocatalytic oxidation of methanol. J. Mater. Sci. 2018, 53, 15871-15881. [CrossRef]

29. Mao, J.; Liu, Y.; Chen, Z.; Wang, D.; Li, Y. Bimetallic Pd-Cu nanocrystals and their tunable catalytic properties. Chem. Commun. 2014, 50, 4588-4591. [CrossRef]

30. Guo, W.; Jiao, J.; Tian, K.; Tang, Y.; Jia, Y.; Li, R.; Wang, H. Controllable synthesis of core-satellites $\mathrm{Fe}_{3} \mathrm{O}_{4} @$ Polypyrrole/Pd nanoarchitectures with aggregation-free Pd nanocrystals confined into Polypyrrole satellite as magnetically recoverable and highly efficient heterogeneous catalysts. RSC Adv. 2015, 5, 102210-102218. [CrossRef]

31. Yin, J.; Zhang, L.; Jiao, T.; Zou, G.; Bai, Z.; Chen, Y.; Peng, Q. Highly Efficient Catalytic Performances of Nitro Compounds and Morin via Self-Assembled MXene-Pd Nanocomposites Synthesized through Self-Reduction Strategy. Nanomaterials 2019, 9, 1009. [CrossRef] [PubMed]

32. Bakshi, M.S. How surfactants control crystal growth of nanomaterials. Cryst. Growth. Des. 2015, 16, 1104-1133. [CrossRef]

33. Niu, W.; Zhang, L.; Xu, G. Shape-controlled synthesis of single-crystalline palladium nanocrystals. ACS Nano 2010, 4, 1987-1996. [CrossRef] [PubMed]

34. Yazdankhah, M.; Veisi, H.; Hemmati, S. In situ immobilized palladium nanoparticles (Pd NPs) on fritillariaimperialis flower extract-modified graphene and their catalytic activity for reduction of 4-nitrophenol. J. Taiwan Inst. Chem. Eng. 2018, 91, 38-46. [CrossRef]

35. Guo, Y.; Xu, Y.T.; Gao, G.H.; Wang, T.; Zhao, B.; Fu, X.Z.; Wong, C.P. Electro-oxidation of formaldehyde and methanol over hollow porous palladium nanoparticles with enhanced catalytic activity. Catal. Commun. 2015, 58, 40-45. [CrossRef]

36. Wang, C.; Sun, S.; Zhang, L.; Yin, J.; Jiao, T.; Zhang, L.; Xu, Y.; Zhou, J.; Peng, Q. Facile preparation and catalytic performance characterization of AuNPs-loaded hierarchical electrospun composite fibers by solvent vapor annealing treatment. Colloid Surf. A 2019, 561, 283-291. [CrossRef]

37. Ren, M.; Zou, L.; Yuan, T.; Huang, Q.; Zou, Z.; Li, X.; Yang, H. Novel palladium flower-like nanostructured networks for electrocatalytic oxidation of formic acid. J. Power Sources 2014, 267, 527-532. [CrossRef]

38. Xiong, Y.; Chen, J.; Wiley, B.; Xia, Y.; Yin, Y.; Li, Z.Y. Size-dependence of surface plasmon resonance and oxidation for Pd nanocubes synthesized via a seed etching process. Nano Lett. 2005, 5, 1237-1242. [CrossRef]

39. Xiong, Y.; McLellan, J.M.; Yin, Y.; Xia, Y. Synthesis of palladium icosahedra with twinned structure by blocking oxidative etching with citric acid or citrate ions. Angew. Chem. Int. Ed. 2007, 46, 790-794. [CrossRef]

40. Berhault, G.; Bausach, M.; Bisson, L.; Becerra, L.; Thomazeau, C.; Uzio, D. Seed-mediated synthesis of Pd nanocrystals: Factors influencing a kinetic-or thermodynamic-controlled growth regime. J. Phys. Chem. C 2007, 111, 5915-5925. [CrossRef]

41. Chang, G.; Oyama, M.; Hirao, K. Facile synthesis of monodisperse palladium nanocubes and the characteristics of self-assembly. Acta Mater. 2007, 55, 3453-3456. [CrossRef]

42. Zhu, W.; Zhang, L.; Yang, P.; Chang, X.; Dong, H.; Li, A.; Gong, J. Morphological and compositional design of $\mathrm{Pd}-\mathrm{Cu}$ bimetallic nanocatalysts with controllable product selectivity toward $\mathrm{CO}_{2}$ electroreduction. Small 2018, 14, 1703314. [CrossRef]

43. Luo, X.; Ma, K.; Jiao, T.; Xing, R.; Zhang, L.; Zhou, J.; Li, B. Graphene oxide-polymer composite Langmuir films constructed by interfacial thiol-ene photopolymerization. Nanoscale Res. Let. 2017, 12, 99. [CrossRef] [PubMed]

44. Zhang, P.; Song, T.; Wang, T.; Zeng, H. In-situ synthesis of Cu nanoparticles hybridized with carbon quantum dots as a broad spectrum photocatalyst for improvement of photocatalytic $\mathrm{H}_{2}$ evolution. Appl. Catal. B 2017, 206, 328-335. [CrossRef]

45. Kunz, M.R.; McClain, S.M.; Chen, D.P.; Koczkur, K.M.; Weiner, R.G.; Skrabalak, S.E. Seed-mediated co-reduction in a large lattice mismatch system: Synthesis of Pd-Cu nanostructures. Nanoscale 2017, 9, 7570-7576. [CrossRef] [PubMed]

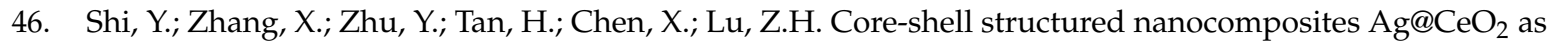
catalysts for hydrogenation of 4-nitrophenol and 2-nitroaniline. RSC Adv. 2016, 6, 47966-47973. [CrossRef] 
47. Baghbamidi, S.E.; Hassankhani, A.; Sanchooli, E.; Sadeghzadeh, S.M. The reduction of 4-nitrophenol and 2-nitroaniline by palladium catalyst based on a KCC-1/IL in aqueous solution. Appl. Organomet. Chem. 2018, 32, e4251. [CrossRef]

48. Zhao, X.; Jiao, T.; Xing, R.; Huang, H.; Hu, J.; Qu, Y.; Peng, Q. Preparation of diamond-based AuNP-modified nanocomposites with elevated catalytic performances. RSC Adv. 2017, 7, 49923-49930. [CrossRef]

49. Zhao, X.; Ma, K.; Jiao, T.; Xing, R.; Ma, X.; Hu, J.; Yan, X. Fabrication of hierarchical layer-by-layer assembled diamond-based core-shell nanocomposites as highly efficient dye absorbents for wastewater treatment. Sci. Rep. 2017, 7, 44076. [CrossRef]

50. Yin, J.; Zhan, F.; Jiao, T.; Deng, H.; Zou, G.; Bai, Z.; Zhang, Q.; Peng, Q. Highly efficient catalytic performances of nitro compounds via hierarchical PdNPs-loaded MXene/polymer nanocomposites synthesized through electrospinning strategy for wastewater treatment. Chin. Chem. Lett. 2019. [CrossRef]

51. Zhao, J.; Yin, J.; Zhong, J.; Jiao, T.; Bai, Z.; Wang, S.; Zhang, L.; Peng, Q. Facile preparation of a self-assembled artemia cyst shell- $\mathrm{TiO}_{2}-\mathrm{MoS}_{2}$ porous composite structure with highly efficient catalytic reduction of nitro compounds for wastewater treatment. Nanotechnology 2020, 31, 085603. [CrossRef]

52. Zhu, J.; Wang, R.; Geng, R.; Zhang, X.; Wang, F.; Jiao, T.; Yang, J.; Bai, Z.; Peng, Q. A facile preparation method for new two-component supramolecular hydrogels and their performances in adsorption, catalysis, and stimuli-response. RSC Adv. 2019, 9, 22551-22558. [CrossRef]

53. Wang, C.; Yin, J.; Han, S.; Jiao, T.; Bai, Z.; Zhou, J.; Zhang, L.; Peng, Q. Preparation of Palladium Nanoparticles Decorated Polyethyleneimine/Polycaprolactone Composite Fibers Constructed by Electrospinning with Highly Efficient and Recyclable Catalytic Performances. Catalysts 2019, 9, 559. [CrossRef]

54. Jin, Q.; Chen, S.; Sang, Y.; Guo, H.; Dong, S.; Han, J.; Chen, W.; Yang, X.; Li, F.; Duan, P. Circularly polarized luminescence of achiral open-shell pi-radicals. Chem. Commun. 2019, 55, 6583-6586. [CrossRef] [PubMed]

55. Jin, Q.; Chen, S.; Jiang, H.; Wang, Y.; Zhang, L.; Liu, M. Self-Assembly of Amphiphilic Schiff Base and Selectively Turn on Circularly Polarized Luminescence by $\mathrm{Al}^{3+}$. Langmuir 2018, 34, 14402-14409. [CrossRef] [PubMed]

56. Zhang, Y.; Yang, D.; Han, J.; Zhou, J.; Jin, Q.; Liu, M.; Duan, P. Circularly Polarized Luminescence from a Pyrene-Cyclodextrin Supra-Dendron. Langmuir 2018, 34, 5821-5830. [CrossRef] [PubMed]

57. Jin, Q.; Li, J.; Zhang, L.; Fang, S.; Liu, M. Reactive organogels based on isoxazole esters: Alkali metal ions selective gelation and crystallization. Cryst. Eng. Commun. 2015, 17, 8058-8063. [CrossRef]

58. Jin, Q.; Li, J.; Li, X.; Zhang, L.; Fang, S.; Liu, M. Function and Application of Supramolecular Gels Chiral Molecular Recognition and Asymmetric Catalysis. Prog. Chem. 2014, 26, 919-930.

59. Li, H.; Yin, J.; Meng, Y.; Liu, S.; Jiao, T. Nickel/Cobalt-Containing Polypyrrole Hydrogel-Derived Approach for Efficient ORR Electrocatalyst. Colloid Surf. A 2020. [CrossRef]

60. Ma, K.; Wang, R.; Jiao, T.; Zhou, J.; Zhang, L.; Li, J.; Bai, Z.; Peng, Q. Preparation and aggregate state regulation of co-assembly graphene oxide-porphyrin composite Langmuir films via surface-modified graphene oxide sheets. Colloid Surf. A 2020, 584, 124023. [CrossRef]

61. Meng, Y.; Yin, J.; Jiao, T.; Bai, J.; Zhang, L.; Su, J.; Liu, S.; Bai, Z.; Cao, M.; Peng, Q. Self-assembled copper/cobalt-containing polypyrrole hydrogels for highly efficient ORR electrocatalysts. J. Mol. Liq. 2020. [CrossRef]

62. Hou, N.; Wang, R.; Geng, R.; Wang, F.; Jiao, T.; Zhang, L.; Zhou, J.; Bai, Z.; Peng, Q. Facile Preparation of Self-Assembled Hydrogels Constructed by Poly-Cyclodextrin and Poly-Adamantane as Highly Selective Adsorbents for Wastewater Treatment. Soft Matter 2019, 15, 6097-6106. [CrossRef] [PubMed]

63. Ma, K.; Chen, W.; Jiao, T.; Jin, X.; Sang, Y.; Yang, D.; Zhou, J.; Liu, M.; Duan, P. Boosting Circularly Polarized Luminescence of Small Organic Molecules via Multi-Dimensional Morphology Control. Chem. Sci. 2019, 10, 6821-6827. [CrossRef] [PubMed]

64. He, Y.; Wang, R.; Jiao, T.; Yan, X.; Wang, M.; Zhang, L.; Bai, Z.; Zhang, Q.; Peng, Q. Facile Preparation of Self-Assembled Layered Double Hydroxide-Based Composite Dye Films as New Chemical Gas Sensors. ACS Sustain. Chem. Eng. 2019, 7, 10888-10899. [CrossRef]

(C) 2019 by the authors. Licensee MDPI, Basel, Switzerland. This article is an open access article distributed under the terms and conditions of the Creative Commons Attribution (CC BY) license (http://creativecommons.org/licenses/by/4.0/). 